\title{
SmartCoM: Smart Consumption Management Architecture for Providing a User-Friendly Smart Home based on Metering and Computational Intelligence
}

\author{
Edvar da L. Oliveira, Rodrigo D. Alfaia, Anderson V. F. Souto, Marcelino S. Silva, Carlos Renato L. \\ Francês \\ Federal University of Pará (UFPA), Belém, Pará,Brazil, ZIP:66075-110,E-mail: edvar@ufpa.br, \\ rodrigo.alfaia@itec.ufpa.br, anderson.souto@itec.ufpa.br, marcelino@ufpa.br, rfrances@ufpa.br \\ N. L. Vijaykumar \\ National Institute for Space Research (INPE), São José dos Campos, São Paulo, Brazil, ZIP: 12227-010, E- \\ mail:vijay.nl@inpe.br
}

\begin{abstract}
With advances in information technology for health and wellness, Smart Home-based solution providers using Internet of Things (IoT) technologies, have increased in importance and become accepted as an alternative means of saving energy when based on Home Energy Management Systems (HEMS). This paper defines a modern architecture (SmartCoM), which is implemented to monitor and manage residential dwellings by using IoT technologies. This involves setting out the parameters that can make interoperability possible between measurement and management, and the layers of data communication, which are the features necessary for the hardware required for monitoring and measurement. In addition, an interface is defined by a middleware layer to integrate the management of external installations and the visualization of data by means of a cloud service. The SmartCoM end-to-end architecture is defined in detail from the standpoint of the consumer and optimization strategies are employed for both the end customer and the utility. The main advantages of using SmartCoM were confirmed by the numerical results obtained from the proposed architecture. This paper ends by showing the current position of SmartCoM as well as suggesting further stages for this line of research.
\end{abstract}

Index Terms - device software platforms, Internet of Things, middleware, Smart Home.

\section{INTRODUCTION}

In recent years, the panorama of the power systems has been subject to change following the introduction of new business models, for example, electric power distribution. These new models enable consumers to be actively involved on the basis of Smart Grid experiences. This participation makes the scenario more complex with respect to services since it raises serious challenges - for example, when integrating, software and hardware features. This is because the power systems and power companies operate in heterogeneous environments which is an essential strategic factor in ensuring a certain degree of safety (an inherent feature of the electricity sector). As well as this, there is also a need to include services that can outperform those of the traditional electricity networks. 
It is thus advisable to devise a strategy that can be interoperable between all aspects of the electricity sector (with all its diverse nuances). In addition, the new model must comply with the standards of the Electricity Regulatory Authority with regard to supervision, maintenance and control, as well as meeting the following requirements: reliability, scalability, management, generation of renewable and clean sources of energy, interoperability and cost-effectiveness. All these features are specified within the domain of Smart Grid [1].

In general, Smart Grid can be regarded as a network that can manage electrical devices in several domains, and provide efficiency, reliability, safety and quality of services. It has a set of seven interconnected domains [2]. The first four (large-scale generation, transmission, distribution and end consumers or customers) are responsible for generation, transmission and distribution. These domains must ensure bi-directional communication between the customers and Advanced Metering Infrastructure (AMI) to provide a "full controller support" (i.e. data exchange) for all the system domains. The last three (market, operations and service providers) refer to the power management market, power distribution and provision of services.

In the case of the end-user domain in particular, [3] this refers to power management on the customer side, together with control and management of electrical appliances to balance and optimize residential power consumption. This is one of the nine requirements for Smart Grid applications (demand response and energy efficiency), when employed for Smart Home scenario, and is the focal point of this paper.

On the basis of the established scenario for developing applications for Smart Home, a new scheme has been devised that combines information technology solutions, advanced communications and sensor systems to create a wide range of new Smart Home applications allied to Internet of Things (IoT). These solutions must be able to allow remote control, access and monitoring (from the Internet) [4], thus increasing scalability.

In light of this, this paper sets out an innovative architecture for HEMS (Home Energy Management Systems). This is known as Smart Consumption Management Architecture (SmartCoM), and is based on the rules established in [5] for the interoperability and applicability of Smart Grids within the context of Smart Home for IoT technologies.

The architecture is flexible and includes key technological features such as: data security, modularity, interoperability, remote management capability, the possible use of computational intelligence heuristics, flexibility and reusability (outlined in Section 4). In addition, the proposal seeks to integrate factors outside the Smart Home domain, for example Smart Grid. It offers an interoperability layer that includes a bus transport service with the utility via AMI, by means of cloud services, and thus create a single integrated interface system. The proposal is validated by means of a HEMS protocol designed in accordance with the requirements of the proposed architecture and creates a proactive fuzzy-aware home-control system (Figure 1). 


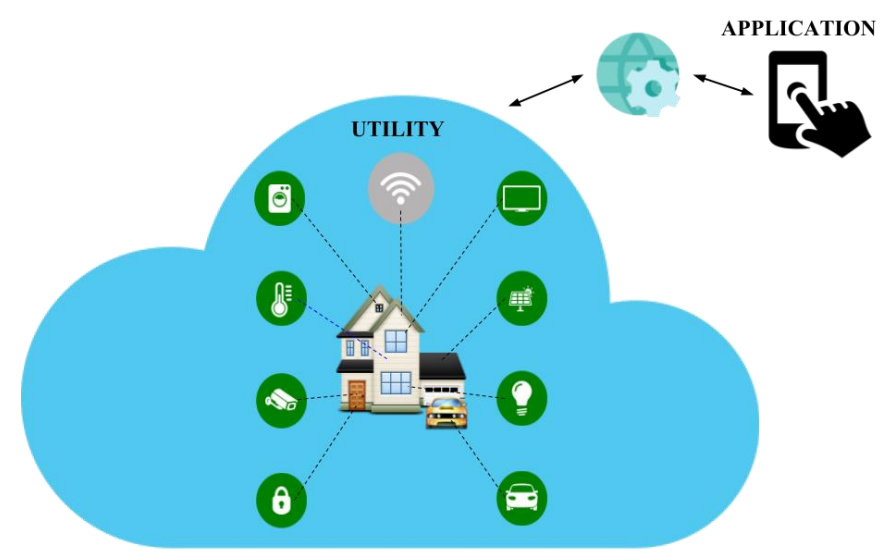

Fig. 1. Conceptual model of the proposed architecture of the IoT for a Smart Home.

\section{RELATED LITERATURE}

Within the general scenario of Smart Home, several HEMS have been proposed to reduce power consumption costs. This reduction is made possible by an exclusive new feature for monitoring and controlling or even adopting computational intelligence solutions to help optimize consumption.

Several investigations have been conducted which involve adopting a single approach to standardize the system needed for the intelligent metering of residential homes. Some are described in [6] and [7], and others are similar to those set out in this paper. A HEMS, that is able to manage household appliances and lighting (by collecting consumption data), is described in [8]. There is an interface with a mobile device that communicates via Zigbee and changes the power distribution of the company to solar panels. The HEMS models are described in [9], [10] and employed to monitor and inform users about the residential consumption rates from mobile applications and the WEB storage system and sensors spread throughout the houses.

Some of the solutions based on computational intelligence in the Smart Home domain are mentioned. Fuzzy logic was employed to adjust the room temperature by means of thermal sensors and patterns were set based on rules recommended by residents to automatically optimize temperature and thus save energy [11]. Similarly, [12] uses fuzzy logic to decide how to schedule the use of appliances on the basis of estimated costs and comfort. In [13], fuzzy is used to reduce the residential energy demand based on different parameters such as occupancy, outdoor temperature, any kind of pricing, thermal comfort, adjusted schedules and preferences in the use of the electricity system

Fuzzy logic is also used for a responsive context-awareness. In [14], fuzzy intelligence shows a 95.14\% degree of accuracy and is $6.98 \mathrm{~ms}$ faster than other architectures.

Other solutions of this kind are used to control and optimize power consumption. One method adapts the Bayesian Network (BN) to control power appliances that are designed for comfort [15]. This network makes a probabilistic relationship between the states of the equipment and the residential environment. 
With regard to IoT, [16] proposed a Smart Home solution, based on Web Services (WS) and Cloud computing. The study employs sensors and actuators to measure the environmental variables and control some of the residential appliances by using Zigbee connections. All the collected data traffic is persisted in the cloud, which establishes the interoperability of the measurement devices, and thus ensures customer have access by means of smartphone or notebook.

In [17], the authors design an architecture that uses the IoT concept to improve the system for monitoring and managing the residential dwelling of the user, through remote access while at the same time, providing more comfort and safety. The user can count on a web or mobile application with access to cloud services, that includes a heuristic system for Smart Home. Another solution for Smart Home is proposed in [18], where a service delivery framework is defined, which relies on IoT for the management of energy consumption.

[19] develops a solution for the management and optimization of electric energy consumption, and improving the security of the homes of users based on IoT. The solution comprises security sensors (fire and presence), relay module for managing the devices, and a central device (connected to the Internet). The interaction between the devices with the user is carried out through Internet, and a mobile application for the management of the residential dwellings.

[20] shows a smart home application based on the Internet concept of things that uses applications for remote access via the Internet. This solution is available for both mobile phones and the laptop with access to a central node. The application can turn home appliances on or off and at the same time record a video captured in live streaming mode of what happens in the environment.

[21] finds a solution for the management of the devices in a residence connected to an IoT network. This adopts an approach to recognize six types of gestures (a fist with one, two, three, four and five fingers) for managing the devices. The operation of the solution is in real time, although it must be configured in two stages: the first to obtain the binary image; the second to recognize the gestures. Its structure is based on ARM Cortex-A8 for data processing and Zigbee is used for communication.

\section{BACKGROUND}

\section{A. Internet of things - IoT}

The Internet of Things (IoT) refers to the network of objects, devices, machines, vehicles, buildings, and other physical systems with embedded sensing, computing, and communication capabilities, that sense and share real-time information about the physical world [22].

By making use of IoT solutions for domestic control, including information and communication technologies, (especially those that ensure high scalability and the availability of services), there can be information sharing and the possibility of remote management of appliances in real time, that will provide access to the users at any time in any place, to all the devices connected in the home.

The IoT is able to connect real world elements and embed the intelligent in communication system 
to smartly process its detailed information and autonomous decision-making, is divided into layers that represent the architecture of operation and management [23]. These layers can be represented as sensors, actuators and collectors in the lower layer, followed by the network layer, which plays an important role in the transfer and security of the information passed to the upper layer. The Middleware Layer is intended to provide data management and storage services for the lower layers. The upper layers are linked to business applications and rules, which are responsible for creating intelligent strategies.

IoT is generally aligned with low power, low memory sensors, and battery and network restrictions, which means there is a need for data processing, storage, access, and analysis [24]. These limitations can be mitigated by the use of other technologies such as the cloud service that will provide processing and memory on demand, as well as different kinds of software that can interact with other applications. As well as this, when IoT is combined with cloud, there are vast amounts of data that can be collected from multiple locations and processed and analyzed to create useful information for the end users.

A cloud computing service is one of the technologies widely used in IoT, which in addition to providing shared resources, storage, software and information, allows external access via any devices connected to the Internet [25-26].

The aim of this study is to find an IoT-based solution that uses cloud computing services, and gives access to all the devices connected to the residence via the Internet, thus enhancing the management and external monitoring of services, such as energy management, security and comfort.

\section{B. Computational intelligence techniques for Decision-Making}

Computational Intelligence (CI) is based on a paradigm to create systems that have some sort of intelligence similar to those present in certain biological systems. The kind of knowledge inspired by computational intelligence modeling has given rise to the following paradigms: i) Symbolic (this consists of a set of symbols to form structures and a set of rules and procedures, for instance Decision Trees); ii) a Connectionist model (this assumes that behavioral intelligence arises from the dynamics of connections between the nodes known as neurons, for instance, Neural Networks); iii) Evolutive (this is based on mechanisms found in nature, that can evolve, such as self-organization and adaptive behavior, for example, Genetic Algorithms); and iv) Fuzzy (this enables the graduation of a certain element belonging to a given class, i.e., it evaluates whether the element belongs to a class with more or less importance, for example, Fuzzy Logic). In our paper, special emphasis is laid on fuzzy systems for decision making, as they are very well established and have been validated for use in applications that involve control, automation, supervision and actuation (more specifically, features that are essential to applications in Smart Grid and Smart Home). Factors, such as the simplicity of the rules, favorable computational effort and results that provide a good deal of support for decision making, enable fuzzy systems to serve as potential alternatives to those systems that have the features 
described in this paper.

Decision-making techniques have been in use for some time to produce results in a natural language and based on the analysis and modeling of complex scenarios. The results are obtained from computational intelligence heuristics. These methods can deal with complex variables, by classifying and/or predicting results to assist decision-making. In this paper, the focus is on fuzzy logic to alert home residents about any increase in energy consumption and, apply an optimization strategy for energy consumption, from the parameterization that occurs at the moment of the SmartCoM configuration.

Fuzzy logic is one of the techniques most widely referred in the specialized literature when dealing with automation. Moreover, it offers the means to interact with processes that are inherently analogous to digital computers [27]. It involves dividing continuous numerical values into a range of states. These states are either overlapping or distorted and defined by using Membership Functions (MF). During the overlapping procedure, the state of a variable does not change all of a sudden. Instead, it gradually loses its value in a MF while renewing it in the next.

The control process starts with fuzzification. At any moment, the system uses the MF of a crisp input variable, which has to be converted into a fuzzy input in which each MF receives a degree of accession. For example, one can convert the input value of the "Consumption Index (Fig. 2) to a diffuse set in which values of "Consumption on Average (C.O.A.), "Consumption Above Average (C.A.A.), "C.A.A. 20\%”, " C.A.A 40\%”, “C.A.A 60\%”, "C.A.A 80\%” and “C.A.A 100\%” receive membership degree 1 for C.O.A, and>1, for consumption within the C.A.A. range.

As a result, a system can then use all the fuzzy sets of the input variables in fuzzy logic. This inference process is based on a set of pre-determined Fuzzy rules that are viewed from the perspective of a specialist, as shown in Figure 2.

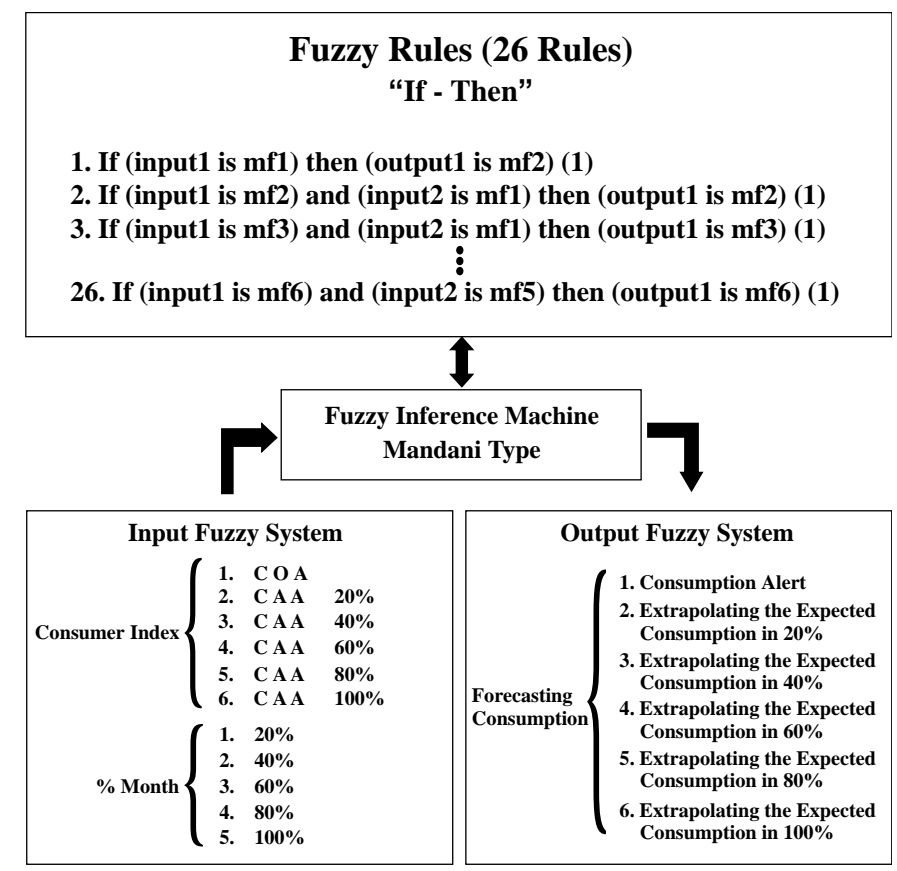

Fig. 2. Fuzzy Inference Machine 
A context is defined by combining all the input values. The system applies relative rules to a certain context. For example, if "Consumption Index" has Membership Degree 1 for "C.O.A." and $>1$ for "C.A.A 20\%", the system checks all the rules containing "C.O.A." or those from "CAA 20\%". All these rules produce fuzzy output values that are combined to create a fuzzy set for each output variable. A variation in the value of the rules is applied so that some rules can be more effective than others. This weight factor is usually between 0 (no effect) and 1 (maximum effect). Finally, the defuzzification phase calculates the final results for the output variables using the obtained fuzzy sets.

The Comfort Index (CI) can be calculated from the output of the fuzzy logic to set the optimization strategy of the energy consumption.

In establishing criteria for a zone of comfort in the relationship between household electrical appliances and human comfort levels, the Comfort Index(CI) formula was conceived as:

$$
\mathrm{CI}=(\mathrm{HEU} / \mathrm{HAH}) * \mathrm{DW}
$$

where HEU (Hours of Equipment Use), HAH (Hours at Home) and DW (Days of the Week).

After this, the application adopts the following strategy (profile):

a) Reduction of Energy Consumption as a Priority Order (Profile 01): The value of excess consumption is subtracted from the amount used by the appliance on which the consumer has least dependence;

The strategy described above takes into account the degree of comfort that each household appliance provides for the user. When seeking to maintain user comforts and ensure there is less impact on the use of equipment, it is possible to help the consumer when his/her daily average rate of energy consumption is exceeded.

\section{SMARTCOM: A PROPOSED ARCHITECTURE}

The proposed architecture offers an innovative interoperability model for Smart Home based on IoT services. It defines the methodology of a REST-based middleware and integrates the supervisory system of the power utility (company) with the metering elements available to the consumer. It is also responsible for controlling alternative energy sources (distributed generation) as well as enabling the automation of domestic appliances by means of intelligent devices and the message control of consumption (for example, rules to schedule routine activities, real-time consumption, effective management of appliances, etc.).

The purpose of the SmartCoM architecture is to guide and assist in the development of applications of interoperability for Smart Home in a transparent, modular, flexible, scalable and reliable way, while respecting the rules established in [28-5], which are the most widely used interoperability solutions in the Smart Home domain. It also establishes the necessary parameters for providing cloud management services. This architecture must be generic so that more efficient solutions can be developed. Details of the features of this architecture are as follows: 
- Transparency: There is no restriction with regard to Access, as long as there is an internet connection and permission granted to the users to manage their monitored appliances regardless of their location;

- Modularity: This is an open architecture and not applicable to the functionalities discussed in the paper. It is possible to extend other management modules (management of lighting, for instance), as long as the communication protocol is maintained;

- Flexibility: The implementation (hardware and software) is not only restricted to the standards listed in this paper. Obviously, the solutions described are those most often referred in the published literature;

- Scalability: It is possible to add new modules as well as to extend the network management;

- Reliability: SmartCom incorporates a procedure for the authentication and security of information at the application level of the internal network, within the standards adopted in this paper;

The architecture is not only concerned with solving the problem of how consumers should carry out their residential management, but also designs a weakly-coupled middleware with publish/subscribe features (IoT services). This enables different interoperability solutions to be found that can support interoperability between different types of middleware, not only for purposes of utility but also for the client, so that communication is possible between systems by exchanging messages; hence the scalability of the system can be increased without being restricted to a single solution.

The proposed architecture also allows functionalities of intelligent management to be adopted, in which heuristics based on computational intelligence can be employed. This fact makes it possible to analyze the variables obtained from the metering equipment and extract patterns to characterize consumption for each consumer so that an optimized model can eventually be applied to Smart Metering. Thus, this architecture not only makes the communication channel feasible between the heterogeneous elements for data traffic, but also extends it so it can be used to analyze and extract patterns of consumption for each monitored end-user.

Figure 3 represents the structural model that underpins the proposed architecture. Each block plays a well-defined role that takes account of the functioning and characteristics of the architecture. It is possible to adapt it to improve scalability and flexibility.

Figure 3 provides a definition of many of the components, which are essential for the reproduction and implementation of the architecture. All the software and hardware components have been defined to be interoperable and to comply with open standards that can be adapted to specific features of a given scenario, communication protocols that are compatible with REST, and both internal (WI-Fi, Bluetooth, etc.) and external (infraestruture of the power utility) communication interfaces. Thus, both the hardware and software components can be customized for users, manufacturers and utilities, since they include the features of interoperability and have adopted open standards when devising the 
architecture.

The cloud and local applications, the central unit and the measurement node, are illustrated in the details of the following diagram:

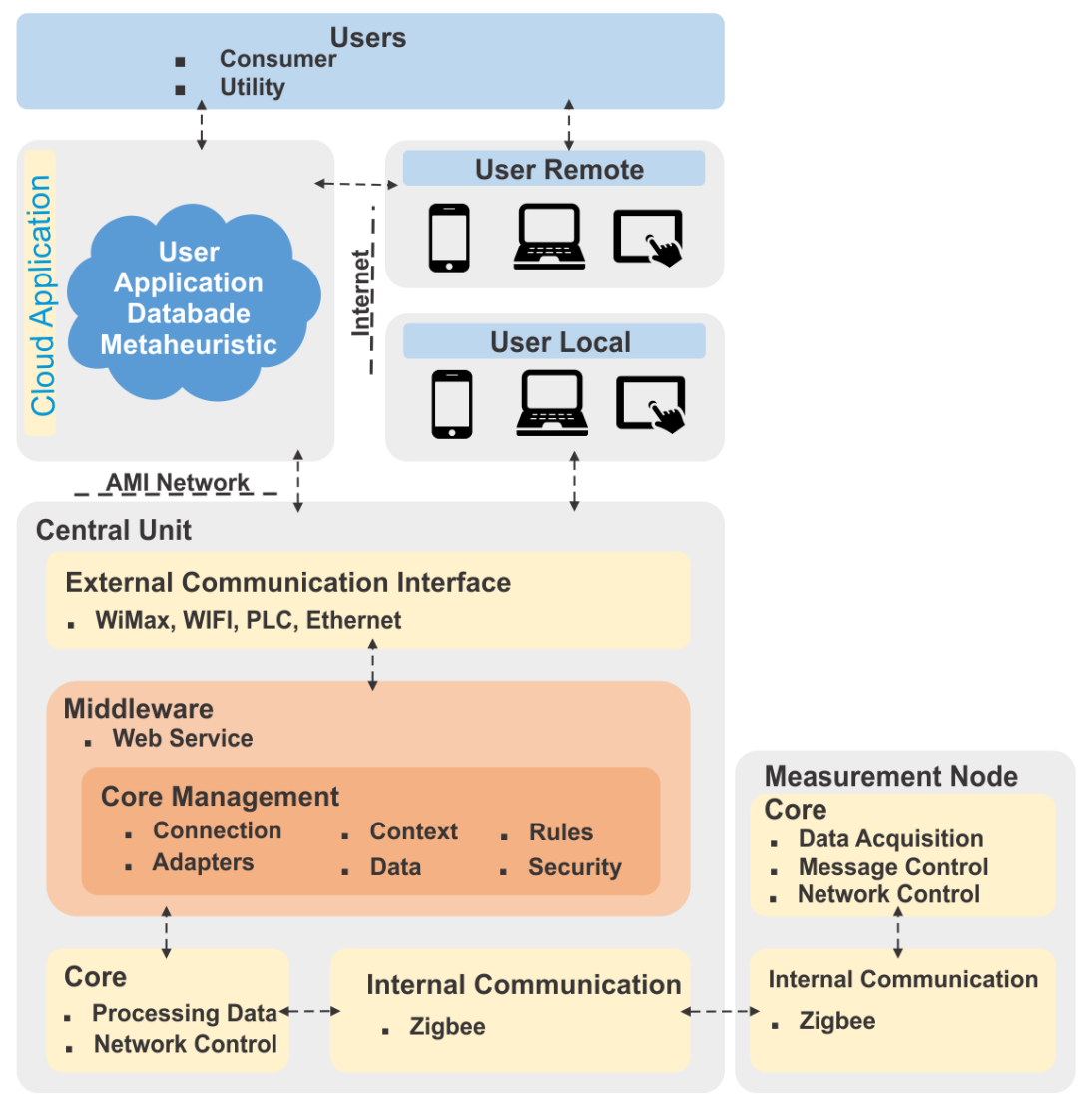

Fig. 3. Diagram of the SmartCoM Architecture

\section{A. Cloud and Local Applications Layers}

The cloud and local applications layers provide mechanisms to monitor and manage elements in accordance with SmartCoM. These applications must be platform-independent and compatible with the REST services.

The cloud layer centralizes the essential services for access and the remote monitoring of the connected elements, since they offer a means of providing information from any device connected to the Internet. This layer specifies the database services that are responsible for storing all the measurement data transferred by the central units. It provides management applications for monitored household appliances, and serve as a mediator in remote management demands.

This layer also specifies the metaheuristic service (computational intelligence techniques) to provide an efficient classification method based on power consumption and its respective management, analysis and establishment of rules for optimization, that can be applied to the metering equipment of each user.

The local and remote applications are responsible for the management of the monitored appliances, using REST requests, they are able to establish a complete control of the available services, on the basis of the access profile. 


\section{B. Central Unit and Measurement Node Layers}

For central unit and measurement node, elements with the communication interfaces are provided to satisfy the communication features specified in the architecture. Integrity is maintained by establishing a dedicated communication channel for the metering elements and thus avoid the loss of information. For example, if there is a bottleneck in the network, a communication interface IEEE 802.15/Zigbee is employed (Internal Communication). This is a wireless network protocol for sensors and low-rate networks, which is widely used to build automation networks such as home security, industrial control and military applications [8]. Moreover, it has the advantage of being able to save energy during a communication.

With regard to the question of aligning it with the standard that is already recommended by Smart Grid, there is a separation between the network (through which the end user requests a service and can make use of open standards such as Wi-Fi, Ethernet and WiMAX), and the network which effectively provides the requested service (in our case, the network utility) with closed standards and potentially less vulnerability, for instance, PLC and 4G). In the case of the central unit in particular, the presence of data communication interfaces for the external environment (the Internet), can help users handle the data. This is described in the architecture as the External Communication Interface.

Both these network access technologies must be transparent and subject to supervisory and monitoring applications. Communication is the key factor for providing data access in heterogeneous domains, including the establishment of real-time access anywhere, and plays an important role in making this architecture functional.

Some management levels are defined as 'core management', such as connection, adapter, context, data, rules and security, among other areas, and require the use of the architecture.

Connection management controls the communication between the supervisor (central unit) and multiple connections of metering devices installed on the consumer side (measurement nodes). This brings the adapter management into the picture so that it can communicate between types of middleware, as the proposal requires a communication interface to be installed by means of WS. Moreover, connection management can preempt traffic and thus allow it to prioritize supervisor messages, when necessary, such as when they are urgently required by the supervisory system of the utility.

Context management plays a role in managing the environment variables of the architecture, and includes the following: communication ports, message priority classes for control and supervision, rules in cryptography, etc. For a more effective and transparent control, it is necessary to identify each appliance monitored by the measurement node.

Each appliance that is registered in the system must have its identification code, and its type if it is essential, so that it can be activated by the monitoring system. This criticality field is crucial importance when formulating and defining rules and strategies for optimizing energy in residential dwellings. Moreover, it also provides a clear overview of the consumption pattern. 
The management of meta-heuristics is necessary for implementing computational intelligence techniques, such as Fuzzy, which is responsible for extracting consumption patterns or creating a set of optimization rules that can be applied in smart devices.

Data management stores and manipulates the data (local and remote), that is obtained from metering devices, environment variables and the remote database (in cloud). In the future, they will also serve to store metaheuristic-based rules (i.e. rules management). This layer must exist for both the supervisor and consumer, as there is no obligation on the part of the manufacturers to provide interfaces to store and manage created rules.

A security management system, from the perspective of the application, is responsible for establishing security criteria for both authentication, transactions, failure recovery, cryptography and access to the services implemented in the platform. As this architecture predicts the use of web services based on REST, the use of security frameworks to guarantee the reliability of information and access is strongly recommended, for example, the OAuth2 framework. Moreover, this layer does not deal with issues such as those that are outdoor related, or the telecommunication network between the providing utility and the end user. However, this paper assumes that security requirements will be met by the network, that is made available by the utility. The specialized literature shows several ways of implementing the telecom network for this objective, for example, as discussed in [5].

The middleware layer generally provides a view of all its components and can be used, for example, to produce control messages such as remotely turn off, and also for consumption adjustment, alternating between energy sources, storing variables of the internal and external environment of each user. In addition, it is responsible for establishing communication with cloud management services. This is an independent module, which is under the supervision of the utility, as a utility uses proprietary tools without any interface to facilitate its integration with other new applications. However, the layer allows management to be carried out by both the client and supervisor by means of REST.

The measurement node specifies the core layer, and is responsible for capturing all the measurements of the monitored appliances (data acquisition), managing the messages of actuation and control (message control) and handling the data sent and received by the network (network control).

\section{IMPLEMENTATION}

A hardware prototype for metering, has been created to validate the system, as well as the necessary software for activating, managing and controlling the residence.

\section{A. Hardware Design}

Apart from all the previously mentioned features, the hardware of the central node must be able to accommodate the installation of the applications (both the middleware and other software needed to store the data) and be able to manage and monitor the measurement nodes, as well as meet the requirements of the end-users. 
A measurement node collects data from the sensors and transmits them to the central node through the Zigbee network. It not only activates the collection unit but also interrupts domestic appliances (i.e. the on/off power). The ideal scenario for an effective monitoring of the residence is that each appliance must have a measurement node connected to it.

The central node also plays a key role in monitoring and managing each connected appliance by collecting data on factors such as the current, tension, consumer power, date and time of metering. All the data is encapsulated in the Zigbee payload and forwarded to the central unit. Its specifications are shown in Figure 4.

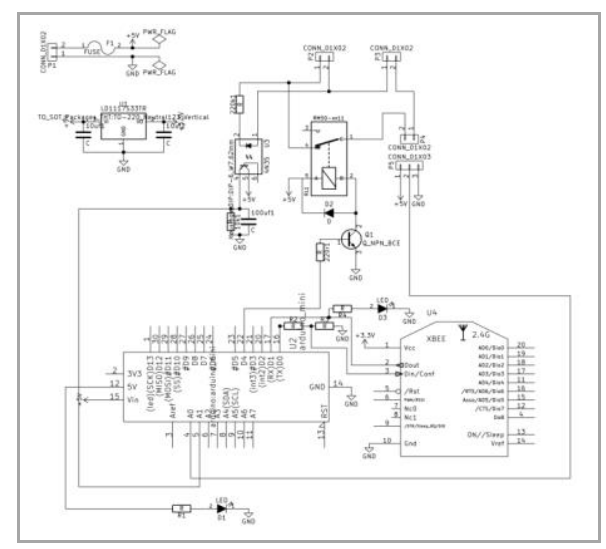

Fig. 4. Structural Diagram of the Measurement node

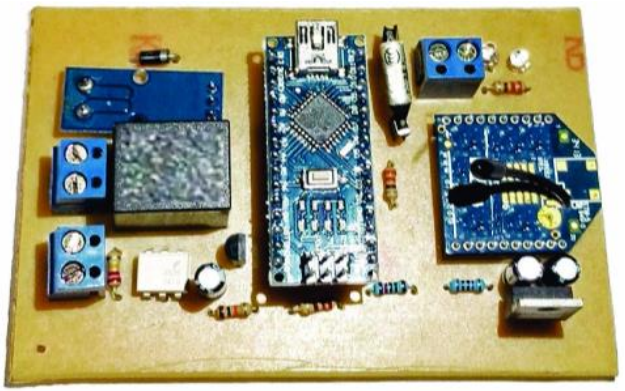

Fig. 5. Prototype of a Measurement node

As shown in Figure 4, the measurement node is initiated by supplying 5v voltage to the entire circuit. After this, the current Hall Effect sensor and tension sensor are used to read the meter and forward the data to the micro-controller. The data is packed and sent via Zigbee to the central node. Similarly, the central node can send a message (of either activation or deactivation) to the end device that may or may not activate a relay on the connected appliance. Figure 5 shows the prototype of the measurement node based on the specifications outlined earlier.

\section{B. Software}

The objective of this paper is to enable interoperability features for Smart Home. For this reason, a service layer has been implemented based on REST that allows interoperability between the metering solution and other external management applications. As a rule, these external applications must be compatible with the WS technology adopted in this paper. Figure 6 shows the components of the middleware solution. 


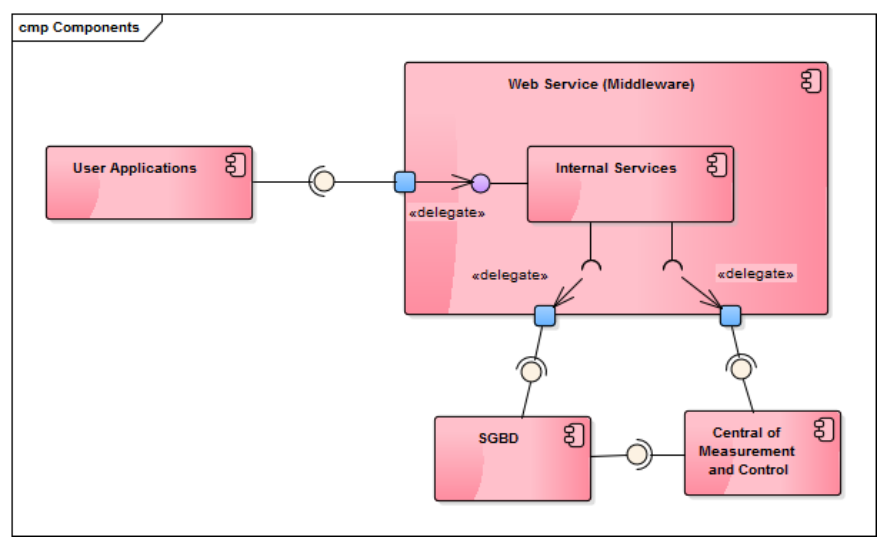

Fig. 6. Diagram with service layer (middleware) components.

The integration uses REST as middleware and includes the features of the above-mentioned technologies. This middleware has an interface to communicate end-user applications (consumer and utility systems), together with residential metering equipment. Moreover, by using this bus, it is possible to carry out several management and control activities, for example, to consult the metering data stored by the central node in a database and parameterize the transfer of metering data to the supervisory system of the utility.

However, some functions will be limited to the type of user to ensure safety and the individual and restricted control of each appliance monitored by the resident. For example, the utility is not allowed to switch off appliances monitored by the solution. This is solely the task of the resident. On the other hand, the utility can interrupt energy manually, a practice that is currently used.

With regard to implementing a meta-heuristic for validation, a method has been found that is based on fuzzy systems. On the basis of the input variables (consumption index and rates), the system can inform the user of the need to reduce consumption and after that, a data processing will be carried out to ensure energy savings.

The Consumption Index (Ind) is calculated from the data supplied by the automatic residential system. These data include: Consumption up to the current date $(C)$, Date $(D m)$, Historical average (A) and Number of Days in the month $(D r)$. On the basis of this information, the index is calculated by (2):

$$
\text { Ind }=C /(\operatorname{Dm}(A / D r))
$$

From (1), one can obtain the values for Ind. They are separated by ranges, where $I n d=1$ indicates that the consumption is within the average band. When Ind $>1$, it is divided into ranges of $20 \%$ to $100 \%$,as shown in Fig. 7. 


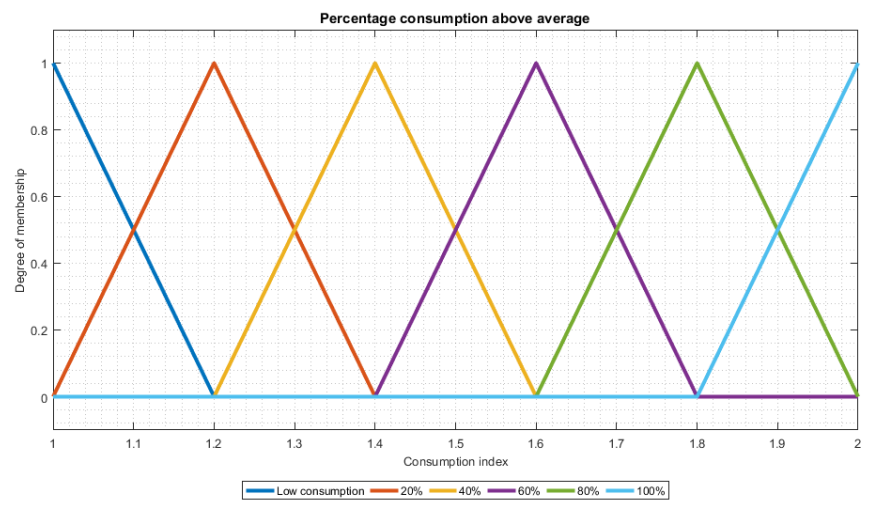

Fig. 7. Consumption Index in Fuzzy Logic.

Likewise, the input variable "\% month" is subdivided into 5 predefined intervals (Fig. 8).

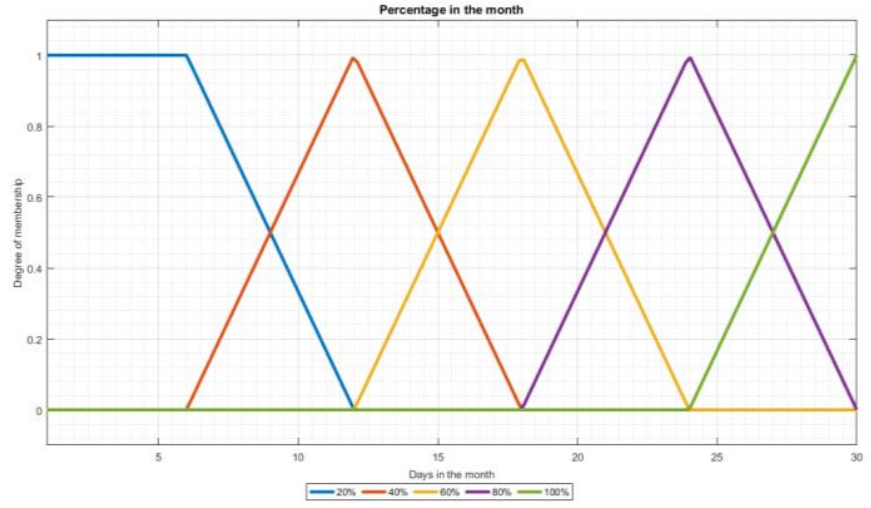

Fig. 8. Input (in \%) of the month in Fuzzy Logic.

After the inference has been made from the Fuzzy machine, the output variable "Exceeded Consumption" (Fig. 9), can assume six possible states starting from the initial point of the diagram, which means that consumption is within the average range. If it is within the range of $20 \%$ to $100 \%$, it means the Consumption level has been exceeded.

In addition, once the Fuzzy system outputs the result, the resident will be notified by an alert in the mobile application. The notification will also list any appliances that exceeded the expected rate of consumption. As a result, the user can take action to ensure a better and more efficient use.

The output of the fuzzy system intrinsically depends on its input variables "Index" and "\% per month". When the input values are changed, the output is also affected.

On the basis of the output, the configuration management system can enable decision-making to be carried out with greater precision by checking the intelligent consumption of the electronic devices so that the user can be notified of the present status of energy consumption.

In addition, the factors that lead to a greater use of energy can be listed from the monitored data of each appliance, in the face of the excessive consumption shown by the fuzzy system. This means that the user can decide which elements should be turned off or reduce its use, on the basis of the defined strategies, and thus optimize energy consumption. Figure 10 shows the flowchart for the decisionmaking system. 


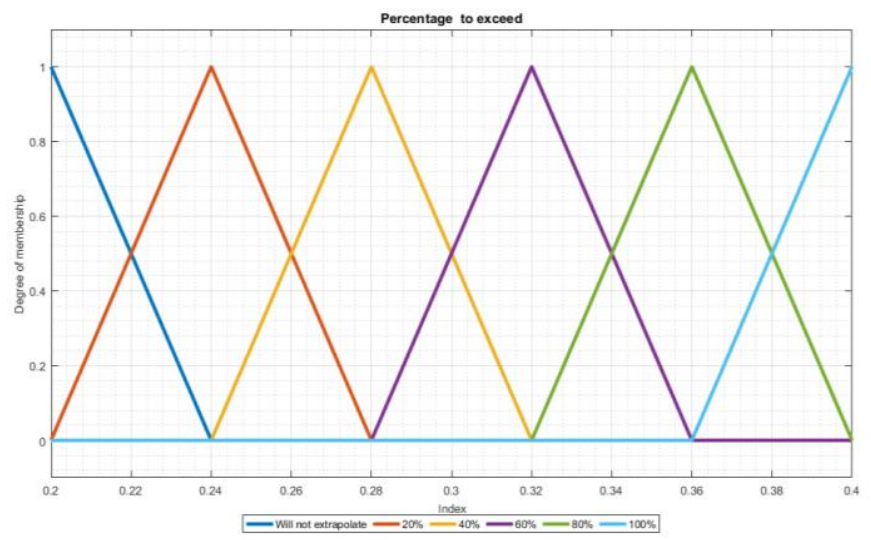

Fig. 9. Output of Exceeded Consumption

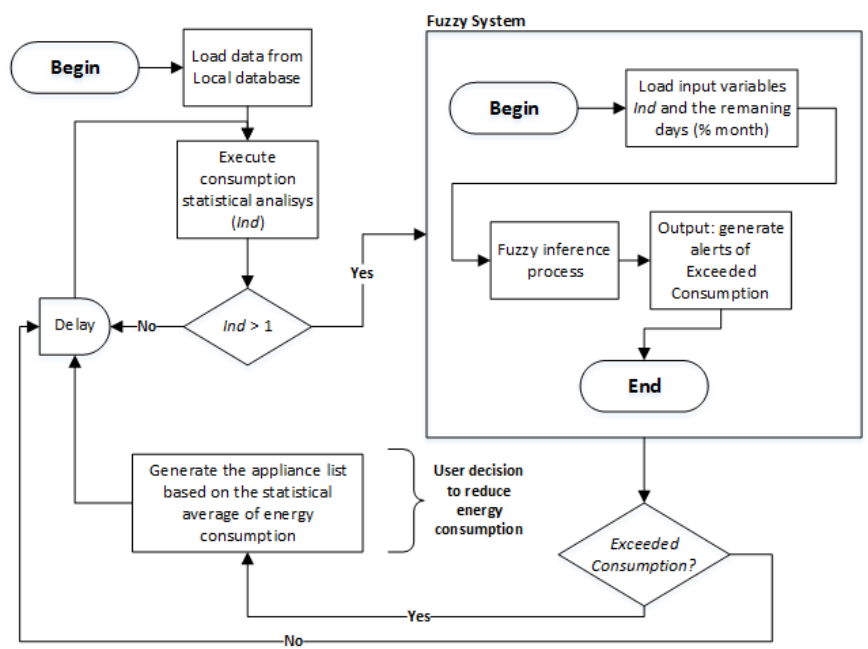

Fig. 10. Flowchart of the decision-making system

\section{VI. (DEMONSTRATION AND) RESULTS WITH A CASE STUDY}

Two systems in different domains were designed for the architecture, one for WEB and the other for mobile devices. The purpose of these two systems is to demonstrate the feasibility of the proposed architecture and its validation.

In both cases, the designed prototypes request management and monitoring services that are in accordance with those of SmartCoM. They are neither restricted to the access platform of the users nor to the established types. Their access restrictions are defined in the authentication scheme. Figures 11 and 12 show the web and mobile case study. 
Journal of Microwaves, Optoelectronics and Electromagnetic Applications, Vol. 16, No. 3, September 2017751 DOI: http://dx.doi.org/10.1590/2179-10742017v16i3965

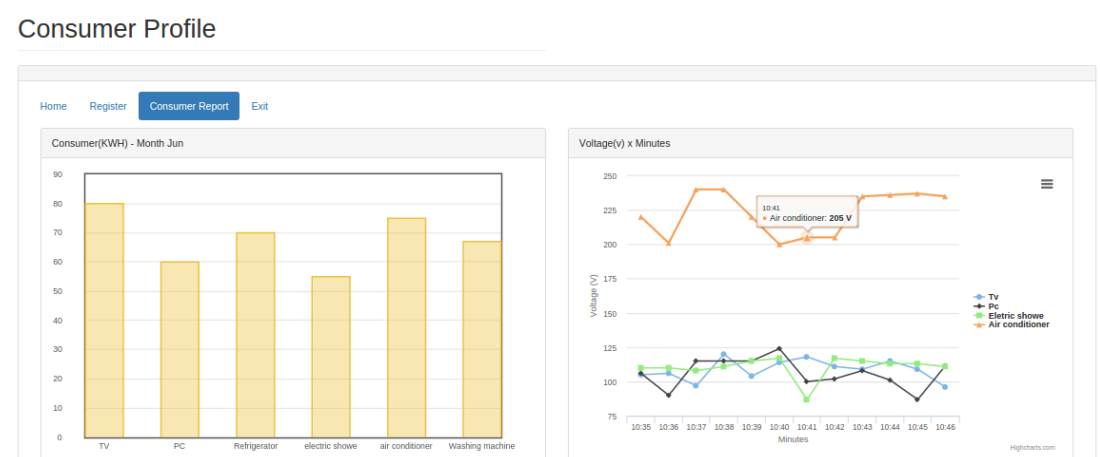

Fig. 11. Monitoring System - WEB case study

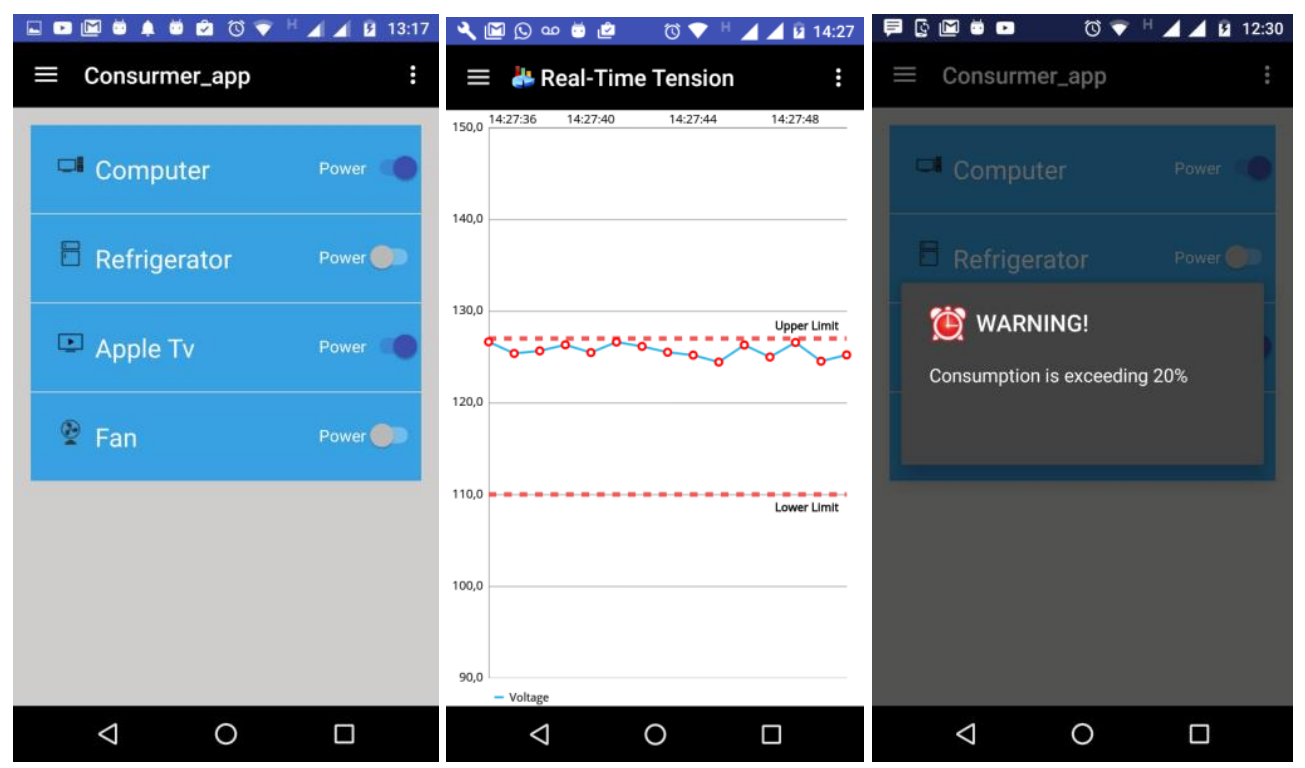

Fig. 12. Mobile Application- (a) Appliance Management Screen (b) Tension Visualization Screen (c) Fuzzy alert

As well as being able to visualize the data by means of the developed solutions, from either locally stored data (but only those of the metering month in the central device) or remotely stored data (utility), it is also possible to obtain other results by manipulating the collected set. Figure 13 illustrates the level of consumption of the monitored equipment.

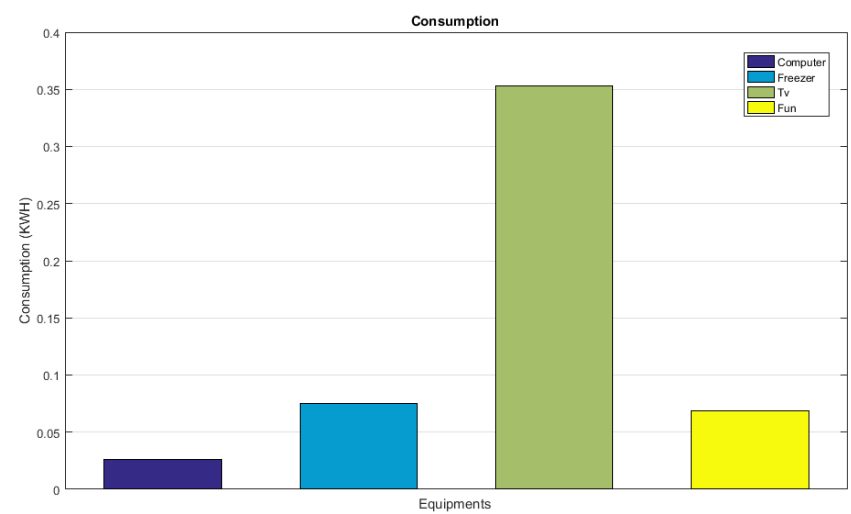

Fig. 13. Chart for Visualizing Consumption (kWh) 
Likewise, it is also possible to handle the stored data of electrical quantities, such as tension and current, as shown in Figures 14 and 15 below.
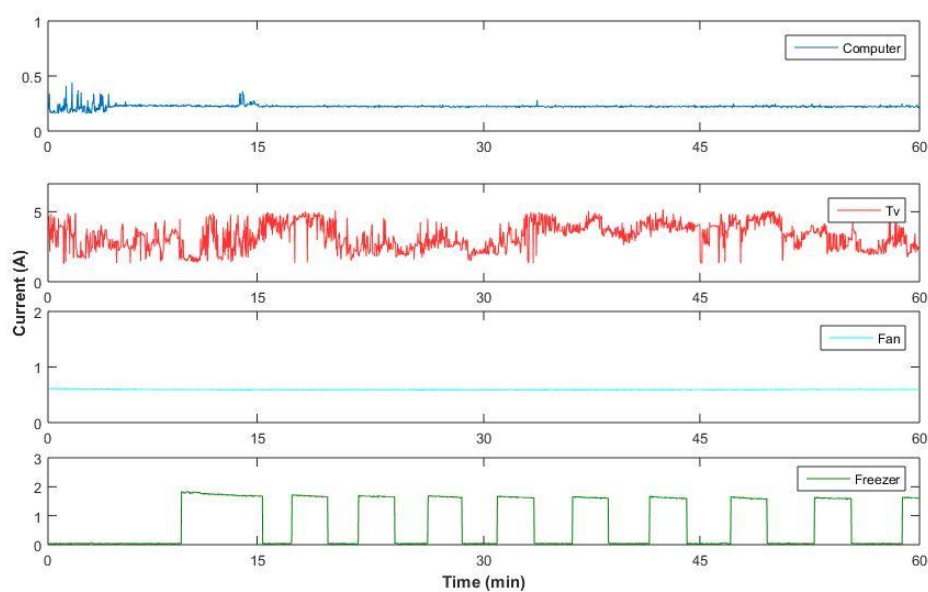

Fig. 14. Current Visualization

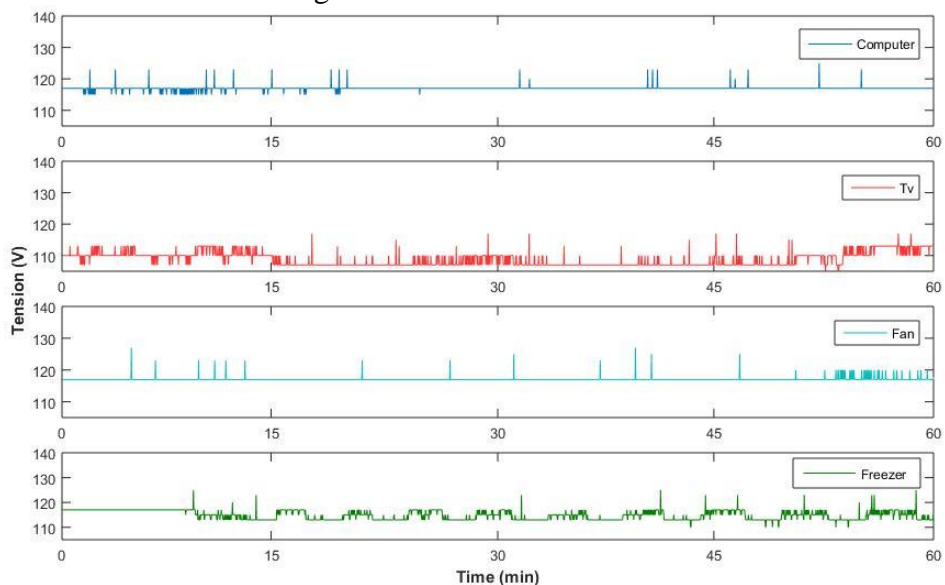

Fig. 15. Tension Visualization

Surveys and measurements were first carried out for testing the intelligent systems. These involved collecting the input data required for the validation of the outputs, which represent the potential for optimizing energy consumption.

On the basis of the collected data (Fig. 16), the intelligent system was employed to evaluate its potential outputs for optimizing energy consumption.

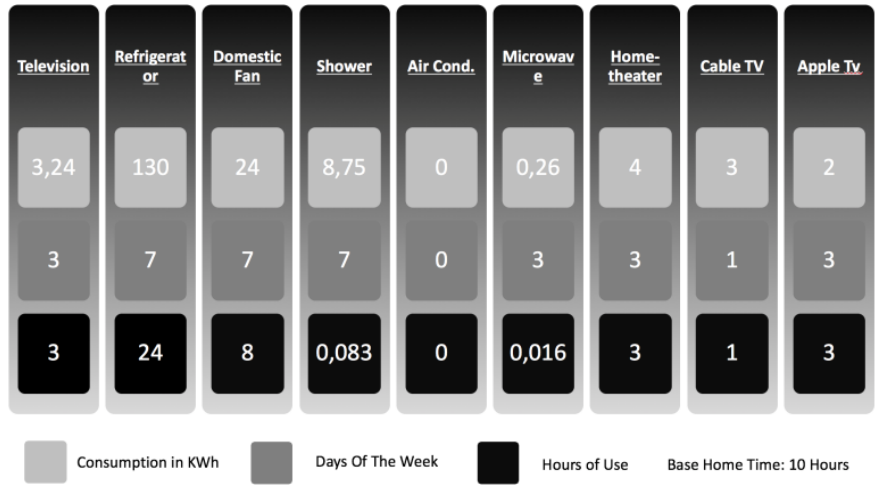

Fig. 16. Average usage and consumption

As a result of the choice of Profile 01 (Fig. 17), it was possible to reduce the energy consumption of 
the last item in the priority list until its excessive rate of energy consumption had been reduced. This was the case with the microwave that previously had a rate of consumption of $5 \mathrm{KWh}$, after the consumption control devices, began to be turned off. The shower previously had a consumption rate of $15 \mathrm{KWh}$ but after the regulatory measures for consumption had been implemented, this was reduced to zero. The rate of consumption for Air Conditioning was already at $0 \mathrm{KWh}$, so the system withdrew consumption from the next item on the priority list, in this case the microwave.

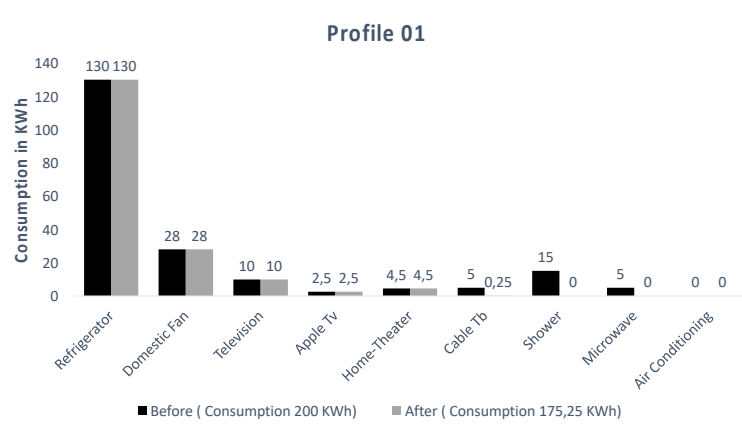

Fig. 17. Equipment Turned Off by Priority Order

A mobile system was designed for the architecture. The purpose of this system is to demonstrate the feasibility of the proposed architecture and its validation. Figure 18 shows the results for energy and time after the profile technique.

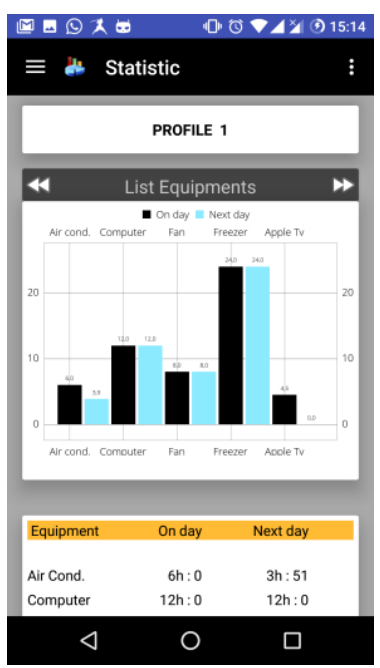

Fig. 18. Results of the Profile Technique.

\section{CONCLUSION}

The main objective of SmartCoM is to find a complete end-to-end solution for Smart Home, on the basis of concepts from device design, metering, interoperability, IoT, computational intelligence, and user-friendly applications. The implementation of a SmartCoM architecture led to the following: a hardware solution for monitoring and a middleware that can provide an interoperable service bus for user-friendly applications aimed at the management module. The architecture also takes into account 
a number of technological features, such as: availability of information, service transparency, modularity, and a high degree of flexibility and reusability.

SmartCoM architecture was the focal point of the essential aspects of the Smart Home domain. Its characteristics and particular features are not only appropriate for the context of monitoring and managing but are also able to provide an interface for management by means of monitoring systems for legacy tools derived from the utility. However, this does not mean that one cannot find one's own solutions to monitor and manage the residences.

It is worth mentioning that the generated data obtained from SmartCoM will make it possible to devise efficient mechanisms that represent an effective means of implementing public policies for reducing power consumption. As can be noted, by employing SmartCoM, it is possible to trace the consumption pattern of each residential customer that is connected. The system can also be beneficial to the environment by employing effective computational intelligence techniques to produce indicators that can assist in reducing the waste of electric power.

The SmartCoM architecture provides a large number of benefits for the Smart Grids environment, in particular, for Smart Home, and these are listed below:

- Development of low-cost open-standards for metering and transmission devices;

- $\quad$ An intelligent module for the classification and prediction of consumption;

- A user-friendly interface for mobile devices to monitor and control the devices of the end-user;

- A Planning and Management consumption system for both the end- user and utilities.

All of these devices and software components are scalable and highly interoperable. They are all based on open standards and a key feature is their low cost. These factors make the SmartCoM architecture suitable for commercial exploitation on a large scale. In addition, the architecture has undergone validation in real-world environments and thus is suitable for use by the utilities.

\section{ACKNOWLEDGMENT}

We would like to the thank the Coordination for the Improvement of Higher Education Personnel (CAPES) and National Council for Scientific and Technological Development - (CNPq), for its financial support.

\section{REFERENCES}

[1] N. Komninos, E. Philippou, and A. Pitsillides, "Survey in Smart Grid and Smart Home Security: Issues, Challenges andCountermeasures," IEEE Commun. Surv. Tutorials, vol. 16, no. 4, pp. 1933-1954, 2014.

[2] Nist, N. S. Publication, and National Institute of Standards and Technology, "NIST Special Publication 1108 NIST Framework and Roadmap for Smart Grid Interoperability Standards," Nist Spec. Publ., vol. 0, pp. 1-90, Oct. 2010.

[3] T. L. Nelson and G. J. FitzPatrick, "NIST role in the interoperable Smart Grid," in 2011 IEEE Power and Energy Society General Meeting, 2011, pp. 1-3.

[4] B. L. Risteska Stojkoska and K. V. Trivodaliev, "A review of Internet of Things for smart home: Challenges and solutions,” J. Clean. Prod., vol. 140, pp. 1454-1464, 2017.

[5] IEEE Guide for Smart Grid Interoperability of Energy Technology and Information Technology Operation with the Electric Power System (EPS), End-Use Applications, and Loads, IEEE Std 2030-2011, setembro. 2011. 
[6] M. Beaudin and H. Zareipour, "Home energy management systems: A review of modelling and complexity," Renew. Sustain. Energy Rev., vol. 45, pp. 318-335, May 2015.

[7] A. Ahmed, S. Razzaq, A. Khan, and F. Khursheed, "HEMSs and enabled demand response in electricity market: An overview,” Renew. Sustain. Energy Rev., vol. 42, pp. 773-785, 2015.

[8] J. Han et al., "Smart home energy management system including renewable energy based on ZigBee and PLC," IEEE Trans. Consumer Electron., vol. 60, No. 2, pp. 198-202, May. 2014.

[9] H. Lee, W.-K. Park, and I.-W. Lee, "A home energy management system for energy-efficient smart homes," in Computational Science and Computational Intelligence (CSCI), 2014 International Conference on, vol. 2, 2014, pp. $142-145$

[10] T. Gabriele, L. Pantoli, V. Stornelli, D. Chiulli, and M. Muttillo, "Smart power management system for home appliances and wellness based on wireless sensors network and mobile technology," in 2015 XVIII AISEM Annual Conference, 2015, pp. 1-4.

[11] B. Walek, J. Zacek, M. Janosek, and R. Farana, "Adaptive fuzzy control of thermal comfort in smart houses," in Proceedings of the 2014 15th International Carpathian Control Conference (ICCC), 2014, pp. 675-678.

[12] M.-R. Haghifam, A. Mohsenzadeh, and M. H. Shariatkhah, "Applying fuzzy techniques to model customer comfort in a smart home control system," in 22nd International Conference and Exhibition on Electricity Distribution (CIRED 2013), 2013, no. 1164, pp. 1164-1164

[13] A. Keshtkar et al. "Smart residential load reduction via fuzzy logic, wireless sensors, and smart grid incentives." Energy and buildings, v. 104, p. 165-180, 2015.

[14] A. Patel and T. A. Champaneria. "Fuzzy logic based algorithm for Context Awareness in IoT for Smart home environment." In: Region 10 Conference (TENCON), 2016 IEEE. IEEE, 2016. p. 1057-1060.

[15] T. Shoji, W. Hirohashi, Y. Fujimoto, and Y. Hayashi, "Home energy management based on Bayesian network considering resident convenience," in 2014 International Conference on Probabilistic Methods Applied to Power Systems (PMAPS), 2014, pp. 1-6.;

[16] M. Soliman, T. Abiodun, T. Hamouda, J. Zhou, and C. Lung, "Smart home: integrating internet of things with web services and cloud computing," in Proc. IEEE International Conference on Cloud Computing Technology and Science, Bristol, pp. 317 - 320, Dec. 2013.

[17] P. Patel, M. Patel, and V. Panchal, "Home Automation Using Internet of Things,” Imp. J., pp. 1-6, 2016.

[18] X. Li, L. Nie, S. Chen, D. Zhan, and X. Xu, "An IoT Service Framework For Smart Home: Case Study On HEM," IEEE Softw., vol. 32, no. 3, pp. 6-6, 2015.

[19] J. Chhabra, "IoT based Smart Home Design using Power and Security Management," no. Iciccs, pp. 6-10, 2016.

[20] V. Patchava, H. B. Kandala and P. R. A. Babu. "Smart Home Automation technique with Raspberry Pi using IoT." In: Smart Sensors and Systems (IC-SSS), International Conference on. IEEE, 2015. p. 1-4.

[21] M. A. Rashid and X. han. "Gesture control of ZigBee connected smart home Internet of Things." In: Informatics, Electronics and Vision (ICIEV), 2016 5th International Conference on. IEEE, 2016. p. 667-670.

[22] B. Marc et al. "IoT-Cloud Service Optimization in Next Generation Smart Environments." IEEE Journal on Selected Areas in Communications, v. 34, n. 12, pp. 4077-4090, 2016.

[23] S. Kraijak and P. Tuwanut, "A survey on IoT architectures, protocols, applications, security, privacy, real-world implementation and future trends," in proc. of the 11th International Conference on Wireless Communications, Networking and Mobile Computing (WiCOM 2015), September 2015

[24] A. Zaslavsky et al., "Sensing as a service and big data." arXiv preprint arXiv:1301.0159 (2013).

[25] S. Nepal, S. Chen, J. Yao, and D. Thilakanathan, "Diaas: Data integrity as a service in the cloud," in Proceedings of the 2011 IEEE 4th International Conference on Cloud Computing, ser. CLOUD '11. IEEE Computer Society, 2011, pp. $308-315$.

[26] Y. Januzaj et al., "DBMS as a Cloud service: Advantages and Disadvantages." Procedia-Social and Behavioral Sciences 195 (2015): 1851-1859.

[27] A. Keshtkar and S. Arzanpour, "An adaptive fuzzy logic system for residential energy management in smart grid environments,” Appl. Energy, vol. 186, pp. 68-81, 2016.

[28] T. G. A. Council, "GridWise Interoperability context - Setting Framework," GridWise Architecture Council and Battelle Memorial Institute, Mar. 2008. 